\title{
The pattern of thyroiditis in multiple sclerosis: a cross-sectional study in a tertiary care hospital in Egypt
}

\author{
Nearmeen M. Rashad ${ }^{1 *}$ D, Marwa G. Amer ${ }^{2}$, Waleed M. Reda Ashour ${ }^{3}$ and Hassan M. Hassanin ${ }^{1}$
}

\begin{abstract}
Background: Multiple sclerosis (MS) is an inflammatory demyelinating disease of the central nervous system with varied clinical features. Disease-modifying drugs (DMDs) of MS associated with different types of thyroiditis. In this cross-sectional study, we aimed to assess the prevalence of thyroid dysfunction in MS and to investigate the association between DMDs and the risk of thyroiditis in MS. A cross-sectional study included 100 patients with relapsing-remitting multiple sclerosis (RRMS) in relapse, and the diagnosed was according to revised McDonald's criteria 2010.

Results: Our results revealed that the prevalence of thyroiditis was 40\%; autoimmune (34\%) and infective (6\%) among patients with RRMS in relapse and cerebellar symptoms were significantly higher in patients with thyroiditis compared to patients without thyroiditis. Regarding the association between DMDs and thyroiditis, the prevalence of patients treated with interferon-beta-1b was higher in MS patients with thyroiditis compared to MS patients without thyroiditis. However, the prevalence of patients treated with interferon-beta-1a was lower in MS patients with thyroiditis compared to MS patients without thyroiditis. In addition, we found CMV infection was more common in patients treated by interferon beta-1 $\mathrm{b}$ and candida infection was common in patients treated by fingolimod.
\end{abstract}

Conclusions: Thyroiditis is commonly observed in patients with RRMS in relapse and higher prevalence of patients treated with interferon-beta-1b which is commonly associated with thyroiditis and CMV infection; however, candida thyroid infection was common in MS patients treated by fingolimod.

Keywords: RRMS, Thyroiditis, Interferon beta-1b, Fingolimod, DMDs

\section{Background}

Multiple sclerosis (MS) is a chronic inflammatory and neurodegenerative disorder of the central nervous system [1]. MS has variable clinical types: acute fulminate forms, clinically isolated syndrome (CIS), relapsingremitting MS (RRMS), and secondary chronic progressive (SP) MS [2].

Mounting evidence indicates that MS associated with other autoimmune diseases as they have the same

\footnotetext{
* Correspondence: nrashad78@yahoo.com

${ }^{1}$ Internal Medicine Department, Faculty of Medicine, Zagazig University,

Zagazig, Egypt

Full list of author information is available at the end of the article
}

genetic or environmental exposures. In further, as new therapies emerge that raise the risk of autoimmune diseases such as thyroid disease, a growing body of evidence has corroborated that most immune therapies for MS are associated with immunosuppression, both shortterm/intermittent (pulsed, induction) and long-term persistent immunosuppression (chronic, maintenance). Immune suppression medication associated with opportunistic or increased infections [3].

Disease-modifying drugs (DMDs) have been utilized to ameliorate MS condition including intramuscular interferon beta-1a, subcutaneous IFN-b, subcutaneous IFNb$1 \mathrm{~b}$, and fingolimod $[4,5]$. Most of these drugs act on the

\section{Springer Open}

(c) The Author(s). 2020 Open Access This article is licensed under a Creative Commons Attribution 4.0 International License, which permits use, sharing, adaptation, distribution and reproduction in any medium or format, as long as you give appropriate credit to the original author(s) and the source, provide a link to the Creative Commons licence, and indicate if changes were made. The images or other third party material in this article are included in the article's Creative Commons licence, unless indicated otherwise in a credit line to the material. If material is not included in the article's Creative Commons licence and your intended use is not permitted by statutory regulation or exceeds the permitted use, you will need to obtain permission directly from the copyright holder. To view a copy of this licence, visit http://creativecommons.org/licenses/by/4.0/. 
immune system and suppress immune cells so that autoreactive immune cells will be unable to attack the myelin sheaths of neurons [6].

Thyroid dysfunction (TD) frequently occurs as an autoimmune complication of immune reconstitution therapy (IRT), especially in individuals with multiple sclerosis treated with DMDs. Moreover, the immune suppression of DMDs is associated with opportunistic or increased infections. We aimed in this study to assess the prevalence of thyroid dysfunction in MS and to investigate the possible causes of thyroiditis in MS in relation to DMDs.

\section{Methods}

This cross-sectional study enrolled 200 consecutive patients fulfilling the diagnostic criteria for MS according to the revised McDonald's criteria 2010 [7]. The patients were recruited from outpatient clinics of Neurology and Internal Medicine Departments, and 40 age- and sexmatched healthy controls. The MS group were diagnosed with relapsing-remitting multiple sclerosis (during relapse) and were divided into two subgroups according to thyroid autoantibodies. The enrolled patients met all of the following criteria of infectious thyroiditis as painful thyroid palpation, fever, and neck pain, together with laboratory tests indicating acute systemic inflammation and abnormal thyrotropin and free thyroxin levels. The diagnosis of autoimmune thyroiditis was obtained based on clinical findings and positive serum antibodies; antithyroglobulin antibodies (anti-TG) and anti-thyroid peroxidase antibodies (anti-TPO). All subjects were subjected to thorough history taking and complete general and neurological examination.

Exclusion criteria included pregnancy, lactation, heart, liver, renal, or other endocrine diseases. The diagnosis was supported by thyroid ultrasonographic examination with a $7.5-\mathrm{MHz}$ transducer, and brain and spine MRI were done for all included patients. Laboratory tests were conducted in the Microbiology Unit of Clinical Pathology Department.

\section{Ethics approval and consent to participate}

Written informed consent was taken from all of the participants after explaining the details and benefits as well as risks to them. The ethical committee of the Faculties of Medicine approved the current study.

\section{Blood sampling and laboratory tests}

Blood samples were drawn from all subjects after an overnight fast. Serum C-reactive protein (CRP) was measured using automated clinical chemistry analyzer Cobas Integra 400 plus (Roche Diagnostics, Deutschland). Serum TSH, FT3, and FT4 levels were measured using the Cobas e $601^{\circ}$ analyzer (Roche Diagnostics,
Mannheim, Germany) by ECLIA. Measurements of serum anti-TG and anti-TPO levels were done by enzyme-linked immunosorbent assay (ELISA) (Genesis Diagnostics, Little port, UK). Complete blood count was done for patients and control using automated hematology analyzer sysmex KX-21N (Sysmex, America).

Blood cultures were done using Bact/ALERT culture bottles and incubated for 7 days in the automated Bact/ALERT 3D Microbial Detection System (bioMérieux, Inc, Durham, USA). The positive blood culture bottles and other isolated samples were initially grown on blood agar and Sabouraud dextrose agar for 24 to $48 \mathrm{~h}$ at $37^{\circ} \mathrm{C}$.

\section{Identification}

Candida colonies appeared as flat, smooth, and pale offwhite colored and identified by gram stain. Germ tube formation had been tested for the isolated colony to differentiate $C$. albicans against non-albicans candida (NAC). Candida species were identified using matrixassisted laser desorption ionization-time of flight mass spectrometry (MALDI-TOF MS) (bioMérieux, Marcy l'Etoile, France) according to the manufacturer instructions.

\section{Antifungal susceptibility testing}

The susceptibility to antifungal agents was carried out using Vitek 2 system (bioMérieux, Inc, Durham, USA) for yeast (card no ACT/YS07) containing serial twofold dilutions of six antifungal drugs: fluconazole, flucytosine, voriconazole, caspofungin, micafungin, and amphotericin $\mathrm{B}$ were provided by the manufacturer and in accordance with the guidelines.

\section{Detection of cytomegaly virus (CMV) serological markers}

Serological screening of blood samples was performed by detection of anti-CMV IgM and IgG by electrochemiluminescence immunoassay (ECLIA) technique using Cobas e 410 analyzers (Roche Diagnostics, Mannheim, Germany). All assays were performed according to the recommended manufacturers' instructions. A reactive result is a sample/cutoff value $\geq 1$ for CMV-IgG and $>1$ for CMV-IgM.

\section{Statistical analysis}

Statistical analyses were performed using the Statistical Package for the Social Sciences for Windows (version 21.0; SPSS, Chicago, IL, USA). Data were expressed using descriptive statistic (mean \pm standard deviation) and were analyzed; the comparison between two groups with parametric variables was done using independent sample $t$ test $(t)$ and nonparametric variables using Mann-Whitney test $(z)$. Logistic regression analysis was 
performed to determine the variable associated with DMDs. We considered $P$ to be significant at $<0.05$ with a $95 \%$ confidence interval $(\mathrm{CI})$.

\section{Results}

Clinical and laboratory characteristics of patients with MS

This cross-sectional study is conducted on patients with RRMS in relapsing. The prevalence of thyroiditis was $40 \%$ (autoimmune $=34 \%$ and infective $=6 \%$ ) as shown in Fig. 1. As regards the clinical picture of MS, only cerebellar symptoms were significantly higher in patients with thyroiditis compared to patients without thyroiditis. Patients with thyroiditis as expected had significantly higher values of thyroid autoantibody, anti-TPO, and anti-TG, as well as TSH, compared to MS without thyroiditis. Regarding inflammatory markers, WBC count, neutrophil count, and hs-CRP were significantly higher in the thyroiditis group as compared to MS without thyroiditis. On the contrary, patients with thyroiditis had significantly lower levels of FT3, FT4, hemoglobin, and platelet compared to MS without thyroiditis (Table $1, P<0.05$ ).

Regarding the prevalence of DMDs in studied groups, we observed a significantly higher prevalence of MS patients with thyroiditis treated with interferon-beta-1b. However, there was a significantly higher prevalence of MS patients without thyroiditis treated with interferon beta-1a (Table 1, $P<0.05$ ).

Clinical and laboratory characteristics of patients with MS classified according to thyroid function tests

Among the thyroiditis group, the 68 patients had autoimmune and the 12 patients had infective thyroiditis. There were statistically significant increases of anti-TPO, $\mathrm{TSH}$, hemoglobin, and platelet in autoimmune group compared to the infective thyroiditis group $(P<0.05)$.
On the other hand, there were significantly lower values of FT3, FT4, WBC count, neutrophil count, and hs-CRP in autoimmune group to infective thyroiditis group of patients $(P<0.05)$ (Table 2$)$.

Regarding the prevalence of DMDs in the thyroiditis group, we observed a significantly higher prevalence of autoimmune thyroiditis in patients treated with interferon beta-1b. However, there was a significantly higher prevalence of infective thyroiditis in patients treated with fingolimod (Table $2, P<0.05$ ).

\section{Prevalence of microorganisms in infective thyroiditis}

Among infective thyroiditis, 5 patients had CMV, 3 had streptococcal infections, 3 had candida, and only 1 patient had staphylococcal infections in Fig. 2.

\section{The association between DMDs and types of microorganisms}

Regarding the association between DMDs and types of microorganisms among infective thyroiditis groups, we found CMV infection was more common in patients treated by interferon beta- $1 \mathrm{~b}$ and candida infection was common in patients treated by fingolimod (Fig. 2).

\section{The association between DMDs and levels of thyroid autoantibodies}

Among the autoimmune thyroiditis group, we found the levels of anti-TPO and anti-TG were significantly higher in patients treated by interferon beta- $1 \mathrm{~b}$ compared to patients treated by fingolimod (Figs. 3 and 4, respectively).

Logistic regression analysis to determine the variable associated with DMDs in MS patients with thyroiditis We further test our results by a logistic regression test. We observed that FT4, TSH, anti-TPO, anti-TG, WBC

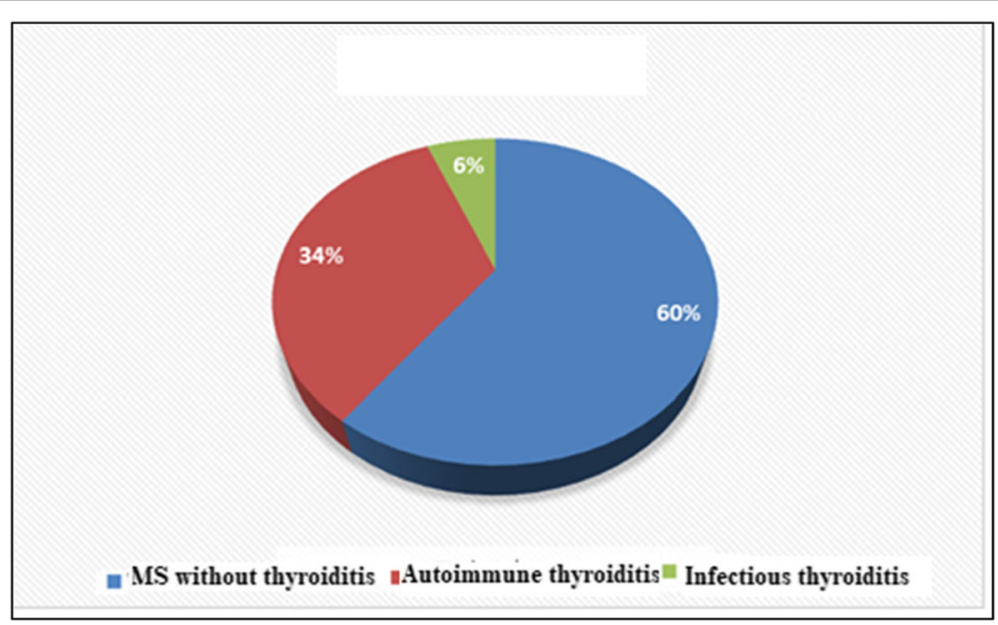

Fig. 1 The prevalence of thyroiditis among patients with multiple sclerosis 
Table 1 Clinical and laboratory characteristics of patients with MS

\begin{tabular}{|c|c|c|c|}
\hline Characteristics & MS without thyroiditis $(n=120)$ & MS with thyroiditis $(n=80)$ & $P$ \\
\hline Age (years) & $30.81 \pm 6.8$ & $30.4 \pm 4.98$ & 0.740 \\
\hline Disease duration/year & $5.97 \pm 0.89$ & $6.45 \pm 0.91$ & $<0.05^{*}$ \\
\hline Number of relapses in the last 2 years & $2.97 \pm 0.92$ & $3.45 \pm 0.93$ & $<0.05^{*}$ \\
\hline \multicolumn{4}{|l|}{ Clinical picture of MS } \\
\hline Sensory & $50(41.7 \%)$ & $46(57.5 \%)$ & 0.089 \\
\hline Motor & $80(66.7 \%)$ & $56(70 \%)$ & 0.450 \\
\hline Cerebellar & $56(46.7 \%)$ & $54(67.5 \%)$ & $<0.05^{*}$ \\
\hline Speech & $16(13.5 \%)$ & $14(17.5 \%)$ & 0.383 \\
\hline Visual & $78(65 \%)$ & $58(72.5 \%)$ & 0.286 \\
\hline Fasting plasma glucose (mg/dl) & $87.3 \pm 4.8$ & $88.5 \pm 3.9$ & 0.732 \\
\hline $\mathrm{FT} 3(\mathrm{pg} / \mathrm{ml})$ & $2.58 \pm 0.32$ & $1.01 \pm 0.79$ & $<0.001^{*}$ \\
\hline $\mathrm{FT} 4(\mathrm{ng} / \mathrm{dl})$ & $1.28 \pm 0.315$ & $1.11 \pm 0.77$ & $<0.001^{*}$ \\
\hline TSH $(\mu \mathrm{lU} / \mathrm{ml})$ & $3.64 \pm 1.76$ & $4.27 \pm 3.420$ & $<0.001^{*}$ \\
\hline Anti-TPO (IU/ml) & $32.64 \pm 8.1$ & $144.87 \pm 55.1$ & $<0.001^{*}$ \\
\hline Anti-TG (IU/ml) & $1.1 \pm 0.2$ & $1.8 \pm 1.52$ & 0.363 \\
\hline WBC count $\left(\right.$ cell $\left.\times 10^{3} / \mu \mathrm{l}\right)$ & $10.3 \pm 1.1$ & $13.7 \pm 6.4$ & $<0.001^{*}$ \\
\hline Hemoglobin (g/dl) & $9.6 \pm 2.71^{\#}$ & $8.5 \pm 1.56$ & $<0.001^{*}$ \\
\hline Platelet $\left(\right.$ cell $\times 10^{3} / \mu$ l) & $114.8 \pm 30.1$ & $77.4 \pm 46.7$ & $<0.001^{*}$ \\
\hline Neutrophil count $\left(\right.$ cell $\left.\times 10^{3} / \mu \mathrm{l}\right)$ & $5.61 \pm 0.72$ & $10.2 \pm 2.68$ & $<0.001^{*}$ \\
\hline hs-CRP ( $\mu \mathrm{g} / \mathrm{ml})$ & $1.16 \pm 0.63$ & $5.94 \pm 3.89$ & $<0.001^{*}$ \\
\hline \multicolumn{4}{|l|}{ DMDs } \\
\hline Methylprednisolone & $60(100 \%)$ & $80(100 \%)$ & - \\
\hline Interferon beta-1a & $18(30 \%)$ & $8(10 \%)$ & $<0.001^{*}$ \\
\hline Interferon beta-1b & $39(65 \%)$ & $60(75.5 \%)$ & $<0.001^{*}$ \\
\hline Fingolimod & $14(23.3 \%)$ & $12(15 \%)$ & 0.224 \\
\hline
\end{tabular}

TSH thyroid-stimulating hormone, FT3 free triidothyronine, FT4 free thyroxine, anti-TG anti-thyroglobulin antibodies, anti-TPO anti-thyroid peroxidase antibodies, $W B C$ white blood cell, $C R P$ C-reactive protein, $D M D$ s disease-modifying drugs $* P<0.05$

count, platelet, neutrophil count, and hs-CRP were significantly associated with DMD (Table $3, P<0.05$ ).

\section{Discussion}

MS is the most prevalent chronic neuroinflammatory disease among young adults and carries the potential risk of permanent disability [8].

Gathering studies have reported the co-occurrence of autoimmune disease with MS, e.g., thyroiditis. As new therapies emerge, the risk of autoimmune diseases rises. We aimed in this study to assess the prevalence of thyroid dysfunction in MS and to investigate the possible causes of thyroiditis in MS in relation to DMDs.

The interesting result of our study was that the prevalence of thyroiditis was $40 \%$, autoimmune $34 \%$, and infective $6 \%$ among patients with RRMS in relapse. The current study revealed that cerebellar symptoms were significantly higher in patients with thyroiditis compared to patients without thyroiditis. Regarding inflammatory markers, WBC count, neutrophil count, and hs-CRP were significantly higher in the thyroiditis group as compared to MS without thyroiditis. Similar to our study, Barone et al. revealed that $12.3 \%$ of patients with MS had thyroid disease [9].

Against our findings, the results of Marrie et al. observed no difference between the prevalence of thyroid dysfunction and anti-thyroid antibodies in MS patients compared with a control population [10].

Numerous immunosuppressant therapies are used for MS treatment. Overall, these agents are safe, have favorable risk-benefit profiles, and can dramatically improve the quality of life for patients with a potentially disabling neurologic illness, but iatrogenic complications were observed. According to the current study, we observed a significantly higher prevalence of MS patients with thyroiditis treated with interferon-beta-1b. However, there was a significantly higher prevalence of MS patients without thyroiditis treated with interferon beta-1a. We 
Table 2 Clinical and laboratory characteristics of patients with MS classified according to thyroid function tests

\begin{tabular}{|c|c|c|c|}
\hline & Autoimmune thyroiditis $(n=68)$ & Infectious thyroiditis $(n=12)$ & $P$ value \\
\hline Age (years) & $29.2 \pm 4.36$ & $31.6 \pm 6.32$ & 0.299 \\
\hline Disease duration/year & $6.3 \pm 0.96$ & $6.7 \pm 0.88$ & 0.215 \\
\hline Number of relapses in the last 2 years & $3.3 \pm 0.98$ & $3.7 \pm 0.87$ & 0.223 \\
\hline \multicolumn{4}{|l|}{ Clinical picture of MS } \\
\hline Sensory & $41(60.2 \%)$ & $7(58.3 \%)$ & 0.580 \\
\hline Motor & $51(76.4 \%)$ & $8(66.7 \%)$ & 0.430 \\
\hline Cerebellar & $52(75 \%)$ & $7(58.3 \%)$ & 0.246 \\
\hline Speech & $10(14.7 \%)$ & $3(25 \%)$ & 0.356 \\
\hline Visual & $51(75 \%)$ & $9(75 \%)$ & 0.645 \\
\hline FT3 (pg/ml) & $1.64 \pm 0.585$ & $2.7 \pm 0.758$ & $<0.001^{*}$ \\
\hline FT4 (ng/dl) & $1.3 \pm 0.57$ & $2.5 \pm 0.75$ & $<0.001^{*}$ \\
\hline TSH $(\mu l U / m l)$ & $6.2 \pm 1.5$ & $0.133 \pm 0.056$ & $<0.001^{*}$ \\
\hline Anti-TPO (IU/ml) & $193.3 \pm 71.08$ & $32.39 \pm 5.76$ & $<0.001^{*}$ \\
\hline Anti-TG (IU/ml) & $1.9 \pm 1.49$ & $1.48 \pm 1.35$ & 0.337 \\
\hline WBC count $\left(\right.$ cell $\left.\times 10^{3} / \mu \mathrm{l}\right)$ & $12.3 \pm 1.9$ & $15.7 \pm 3.4$ & $<0.001^{*}$ \\
\hline Hemoglobin (g/dl) & $8.6 \pm 2.71$ & $8.5 \pm 1.56$ & 0.135 \\
\hline Platelet $\left(\right.$ cell $\left.\times 10^{3} / \mu \mathrm{l}\right)$ & $115.8 \pm 28.1$ & $34.4 \pm 15.1$ & $<0.001^{*}$ \\
\hline Neutrophil count (cell $\left.\times 10^{3} / \mu \mathrm{l}\right)$ & $9.61 \pm 1.091$ & $12.12 \pm 2.68$ & $<0.001^{*}$ \\
\hline hs-CRP ( $\mu \mathrm{g} / \mathrm{ml})$ & $4.46 \pm 1.53$ & $9.64 \pm 2.99$ & $<0.001^{*}$ \\
\hline \multicolumn{4}{|l|}{ DMDs } \\
\hline Methylprednisolone & $68(100 \%)$ & $12(100 \%)$ & - \\
\hline Interferon beta-1a & $6(8.8 \%)$ & $2(16.7 \%)$ & 0.346 \\
\hline Interferon beta-1b & $56(82.3 \%)$ & $4(33.3 \%)$ & $<0.001^{*}$ \\
\hline Fingolimod & $4(5.8 \%)$ & $8(66.6 \%)$ & $<0.001^{*}$ \\
\hline
\end{tabular}

TSH thyroid-stimulating hormone, FT3 free triidothyronine, FT4 free thyroxine, anti-TG anti thyroglobulin antibodies, anti-TPO anti-thyroid peroxidase antibodies, WBC white blood cell, CRP C-reactive protein, DMDs disease-modifying drugs

${ }^{*} P<0.05$

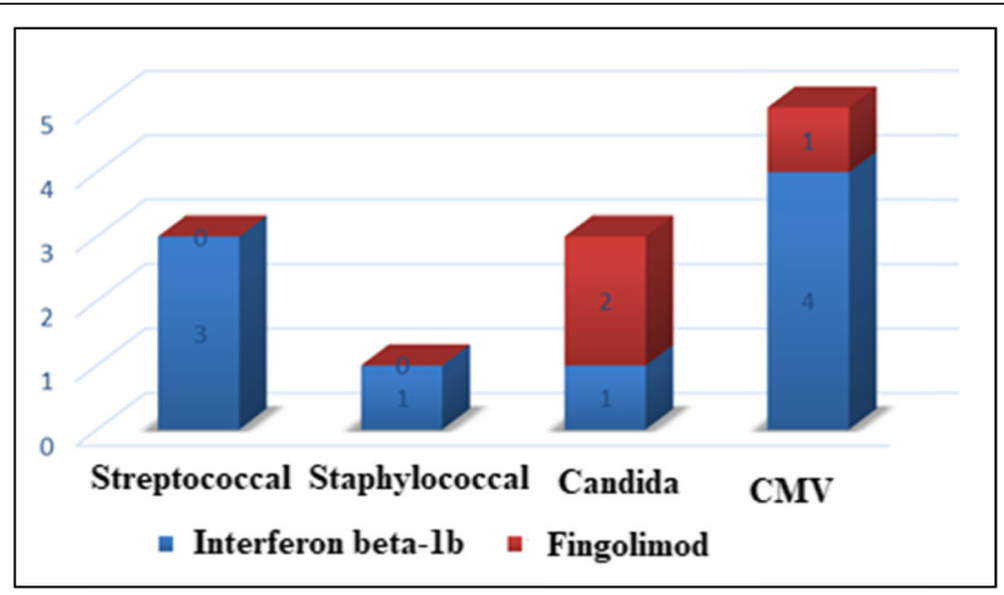

Fig. 2 The prevalence of microorganisms in infective thyroiditis 


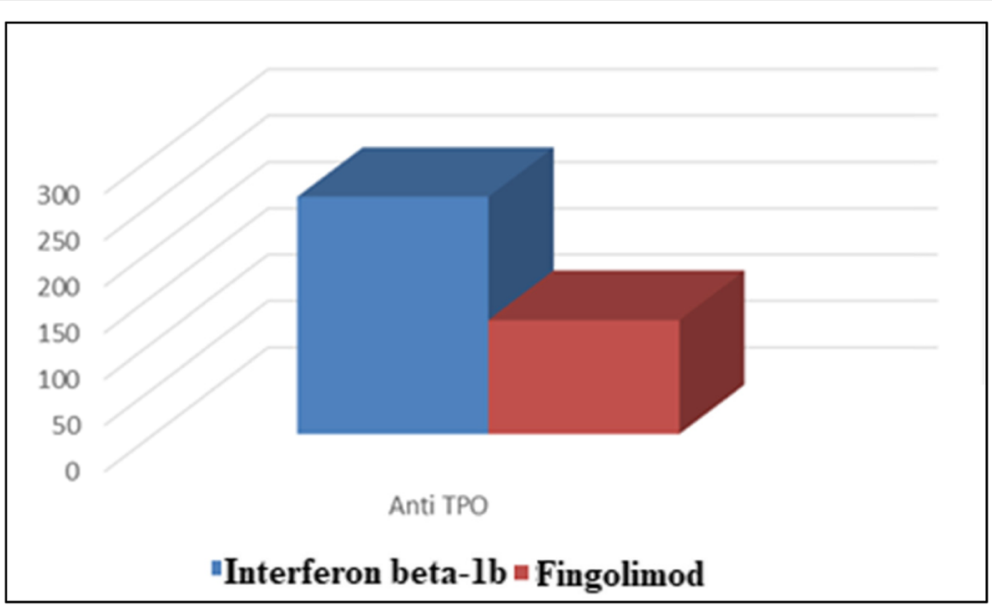

Fig. 3 The association between DMDs and levels of anti-TPO

further test our results by a logistic regression test. We observed that FT3, FT4, TSH, anti-TPO, and anti-TG were significantly associated with interferon beta- $1 \mathrm{~b}$.

Severa et al. reported that IFN- $\beta$ is a key molecule in multiple sclerosis, as it maintains the antiinflammatory status of the immune system and is one of the most widely used treatments for MS. As IFN- $\beta$ modulates the immune-regulatory system, it may precipitate autoimmune disorders. IFN- $\beta$ therapy has been associated with a relatively high risk of developing thyroid disease, either organ dysfunction or autoimmunity [6].

The thyroid gland is a curiously infection-resistant organ because of its high vascularity, lymphatic drainage, tissue uptake of iodine or hydrogen peroxide, and encapsulated structure. Among the factors that may predispose to infective thyroiditis is an immunocompromised status [11]. In the present study, we observed that $4 \%$ of patients with MS had infectious thyroiditis.

The results presented herein are innovative; as this study performs an evaluation of the etiology of infectious thyroiditis in relation to DMDs, we found CMV infection was more common in patients treated by interferon beta- $1 \mathrm{~b}$ and candida infection was common in patients treated by fingolimod.

Jacobs et al. reported that the most common causative organisms of infective thyroiditis are Staphylococci and Streptococci species. Many other organisms such as Acinetobacter, Mycobacterium, Coccidioides, Pseudomonas, Eikenella, Clostridium, Nocardia, Pneumocystis carnie, Haemophilus, and Candida have

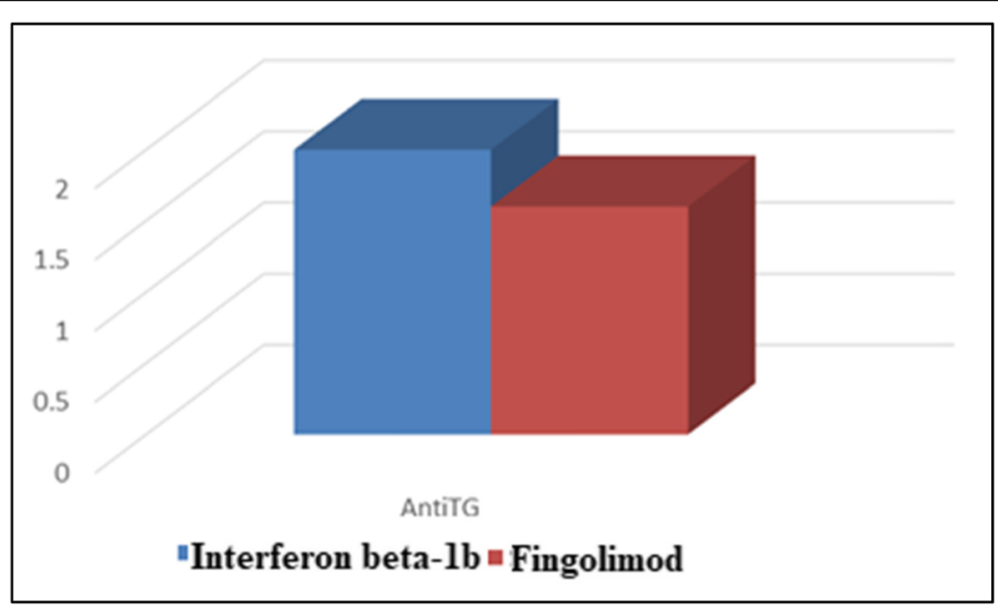

Fig. 4 The association between DMDs and levels of anti-TG 
Table 3 Logistic regression analysis to determine the variable associated with DMDs in MS patients with thyroiditis

\begin{tabular}{|c|c|c|c|c|c|c|c|c|}
\hline \multirow{2}{*}{\multicolumn{2}{|c|}{ Model }} & \multicolumn{2}{|c|}{ Unstandardized coefficients } & \multirow{2}{*}{$\begin{array}{l}\text { Standardized coefficients } \\
\text { Beta }\end{array}$} & \multirow[t]{2}{*}{$t$} & \multirow[t]{2}{*}{ Sig. } & \multicolumn{2}{|c|}{ 95.0\% confidence interval for B } \\
\hline & & B & Std. error & & & & Lower bound & Upper bound \\
\hline \multirow[t]{11}{*}{1} & (Constant) & 0.456 & 0.085 & & 5.368 & $<0.001^{*}$ & 0.287 & 0.625 \\
\hline & FT3 & -0.056 & 0.033 & 0.066 & -1.707 & 0.091 & -0.121 & 0.009 \\
\hline & FT4 & 0.180 & 0.032 & 0.211 & 5.613 & $<0.001^{*}$ & 0.116 & 0.243 \\
\hline & TSH & -0.068 & 0.010 & -0.295 & -6.687 & $<0.001^{*}$ & -0.088 & -0.048 \\
\hline & Anti-TPO & -0.004 & 0.001 & -0.658 & -5.302 & $<0.001^{*}$ & -0.005 & -0.002 \\
\hline & Anti-TG & -0.037 & 0.014 & -0.093 & -2.540 & $<0.05^{*}$ & -0.065 & -0.008 \\
\hline & WBC count & -0.003 & 0.001 & -0.147 & -4.944 & $<0.001^{*}$ & -0.004 & -0.002 \\
\hline & Hemoglobin & -0.017 & 0.010 & -0.075 & -1.741 & 0.085 & -0.037 & 0.002 \\
\hline & Platelet & -0.005 & 0.001 & -0.367 & -7.098 & $<0.001^{*}$ & -0.006 & -0.003 \\
\hline & Neutrophil count & 0.023 & 0.006 & 0.143 & 3.663 & $<0.001^{*}$ & 0.011 & 0.036 \\
\hline & hs-CRP & 0.021 & 0.007 & 0.135 & 2.926 & $<0.05^{*}$ & 0.007 & 0.036 \\
\hline
\end{tabular}

TSH thyroid-stimulating hormone, FT3 free triidothyronine, FT4 free thyroxine, anti-TG anti-thyroglobulin antibodies, anti-TPO anti-thyroid peroxidase antibodies, $T R A b$ thyroid receptor antibody, WBC white blood cell, CRP C-reactive protein, DMDs disease-modifying drugs ${ }^{*} P<0.05$

been isolated, but mostly associated with immunosuppression [12].

Oka et al. detected infection with CMV associated with hyperthyroidism after transplantation due to a reversed imbalance in helper/suppressor $\mathrm{T}$ cell populations and B cell dysregulation [13]. Similar results observed by Maawali et al. regarding the cause of infective thyroiditis could be due to CMV [14].

Chun et al. suggested that fingolimod induced a dosedependent reduction in the peripheral lymphocyte counts to $20-30 \%$ of the baseline value and it also increases the risk of infections [15].

\section{Conclusion}

Thyroiditis is commonly observed in patients with RRMS in relapse and there was higher prevalence of patients treated with interferon-beta- $1 \mathrm{~b}$ which is commonly associated with autoimmune thyroiditis and CMV infection; however, candida thyroid infection was common in MS patients treated by fingolimod.

\section{Abbreviations}

MS: Multiple sclerosis; RRM: Relapsing-remitting multiple sclerosis; DMDs: Disease-modifying drugs; CIS: Clinically isolated syndrome; SP: Secondary chronic progressive; IFN b-1a: Interferon beta-1a; TD: Thyroid dysfunction; IRT: Immune reconstitution therapy; Anti-TG: Globulin antibodies; Anti-TPO: Anti-thyroid peroxidase antibodies; FPG: Fasting plasma glucose; CRP: Serum C-reactive protein; CMV: Cytomegaly virus; TSH: Thyroidstimulating hormone; FT3: Free triiodothyronine; FT4: Free thyroxine; NAC: Non-albicans candida

\section{Acknowledgments}

Not applicable.

\section{Authors' contributions}

NMR, WMRA, and HMH collected the patients' samples and clinical data. MGA prepared the sample for laboratory investigations. NMR wrote the paper. Statistical analysis, interpretation of data, and preparation the paper for submitting international was done by NMR. Critical revision of the manuscript was performed by all of the authors. All the authors have read and approved the manuscript

\section{Funding}

No fund.

\section{Availability of data and materials}

The data that support the findings of this study are available from the corresponding author (nrashad78@yahoo.com) upon reasonable request.

Ethics approval and consent to participate

A written informed consent was taken from all of the participants after explaining the details and benefits as well as risks to them. The ethical committee of Faculties of Medicine, Zagazig University, approved the current study. The reference number is not available yet as there is delay due to the COVID-19 pandemic, but the reference number will be ready as soon as possible.

\section{Consent for publication}

The authors declare that they received consents for publication from all the participants.

\section{Competing interests}

The authors declare that they have no competing interests.

\section{Author details}

${ }^{1}$ Internal Medicine Department, Faculty of Medicine, Zagazig University, Zagazig, Egypt. ${ }^{2}$ Clinical Pathology Department, Faculty of Medicine, Zagazig University, Zagazig, Egypt. ${ }^{3}$ Neurology Department, Faculty of Medicine, Zagazig University, Zagazig, Egypt.

Received: 28 July 2020 Accepted: 24 August 2020

Published online: 27 October 2020

\section{References}

1. Loma I, Heyman R (2011) Multiple sclerosis: pathogenesis and treatment. Curr Neuropharmacol 9(3):409-416

2. Ghasemi N, Razavi S, Nikzad E (2017) Multiple sclerosis: pathogenesis, symptoms, diagnoses and cell-based therapy. Cell J 19(1):1-10. https://doi. org/10.22074/cellj.2016.4867

3. Azizi G, Yazdani R, Rae W (2018) Monogenic polyautoimmunity in primary immunodeficiency diseases. Autoimmun Rev 17:1028-1039

4. Minagar A (2013) Current and future therapies for multiple sclerosis. Scientifica (Cairo) 2013:249101 
5. Golan M, Mausner-Fainberg K, Ibrahim B et al (2019) Fingolimod increases brain-derived neurotrophic factor level secretion from circulating $T$ cells of patients with multiple sclerosis. CNS Drugs 33(12):1229-1237. https://doi. org/10.1007/s40263-019-00675-7

6. Severa M, Rizzo F, Giacomini E (2015) IFN- $\beta$ and multiple sclerosis: crosstalking of immune cells and integration of immunoregulatory networks. Cytokine Growth Factor Rev 26:229-239

7. Polman CH, Reingold SC, Banwell B et al (2011) Diagnostic criteria for multiple sclerosis: 2010 revisions to the McDonald criteria. Ann Neurol 69(2): 292-302

8. Reich DS, Lucchinetti CF, Calabresi PA. Multiple sclerosis. N Engl J Med. 2018;378::169-80.

9. Barone D, Khelemsky S, Hercules D, Barone K. Prevalence of thyroid disease in a multiple sclerosis clinic cohort. April 08, 2014; 82.

10. Marrie RA, Yu BN, Leung S, Elliott L, Warren S et al (2012) The incidence and prevalence of thyroid disease do not differ in the multiple sclerosis and general populations. Neuroepidemiology 39:135-142

11. Wong CH, Watson DA, Wilson JD, Chen GY, Chong GC (2002) Acute thyroiditis caused by Streptococcus agalactiae. Intern Med J 32:195-196

12. Jacobs A, Gros DC, Gradon JD (2003) Thyroid abscess due to Acinetobacter calcoaceticus: case report and review of the causes of and current management strategies of thyroid abscesses. South Med J 96:300-307

13. Oka S, Ono K, Nohgawa M (2019) Cytomegalovirus reactivation triggers the late onset of hyperthyroidism after autologous peripheral blood transplantation. Leukemia Research Reports 11:5-7

14. Al Maawali A, Al Yaarubi S, Al FA (2008) An infant with cytomegalovirusinduced subacute thyroiditis. J Pediatr Endocrinol Metab 21:191-193

15. Chun J, Hartung HP (2010) Mechanism of action of oral fingolimod (FTY720) in multiple sclerosis. Clin Neuropharmacol 33:91-101

\section{Publisher's Note}

Springer Nature remains neutral with regard to jurisdictional claims in published maps and institutional affiliations.

\section{Submit your manuscript to a SpringerOpen ${ }^{\circ}$ journal and benefit from:}

- Convenient online submission

- Rigorous peer review

- Open access: articles freely available online

- High visibility within the field

- Retaining the copyright to your article

Submit your next manuscript at $\boldsymbol{\nabla}$ springeropen.com 